\title{
ON CENTRAL LIMIT AND ITERATED LOGARITHM SUPPLEMENTS TO THE MARTINGALE CONVERGENCE THEOREM
}

\author{
C. C. HEYDE, C.S.I.R.O. Division of Mathematics and Statistics, Canberra
}

\begin{abstract}
Let $\left\{S_{n}, n \geqq 1\right\}$ be a zero, mean square integrable martingale for which $\lim _{n \rightarrow \infty} E S_{n}^{2}<\infty$ so that $S_{n} \rightarrow S_{\infty}$ a.s., say, by the martingale convergence theorem. The paper is principally concerned with obtaining central limit and iterated $\log$ arithm results for $B_{n}\left(S_{n}-S_{\infty}\right)$ where the multipliers $B_{n} \uparrow \infty$ a.s. An example on the Pólya urn scheme is given to illustrate the results.

RATES OF CONVERGENCE: MARTINGALE CONVERGENCE THEOREM; CENTRAL LIMIT THEOREM; ITERATED LOGARITHM LAW; PÓLYA URN SCHEME
\end{abstract}

\section{Introduction}

A serious criticism that can be levelled at probability theory, and with some justification, is that many of the limit results obtained have their relevance obscured by lack of information on the sample sizes necessary for their use to be appropriate. Furthermore, the problem of rates of convergence in general is exacerbated by the hierarchy of limit theory that now exists.

The most basic results in probability theory seem to be those of the form $Z_{n} \stackrel{\text { a.s. }}{\longrightarrow} Z$ as $n \rightarrow \infty$ and the single most powerful tool for establishing results of this kind would appear to be the martingale convergence theorem. However, rate results for the martingale convergence theorem are notably absent from the literature. It is the object of this paper to contribute to the rectification of this deficiency.

The analogy which we shall principally pursue is with the classical strong law of large numbers (SLLN) and the associated central limit theorem (CLT) and law of the iterated logarithm (LIL) which are rate of convergence results about the SLLN. Indeed, if the $X_{i}, i=1,2,3, \cdots$ are independent and identically distributed with $E\left|X_{1}\right|<\infty, E X_{1}=\mu$ and $S_{n}=\sum_{i=1}^{n} X_{i}$, then the SLLN gives $n^{-1} S_{n} \stackrel{\text { a.s. }}{\rightarrow} \mu$ as $n \rightarrow \infty$. If, additionally, $\operatorname{Var} X_{1}=\sigma^{2}<\infty$, then the CLT gives

$$
n^{1 / 2} \sigma^{-1}\left(n^{-1} S_{n}-\mu\right) \stackrel{\text { d }}{\rightarrow} N(0,1)
$$

and the LIL gives

$$
n^{-1} S_{n}-\mu=\zeta(n)\left(2 n^{-1} \log \log n\right)^{1 / 2}
$$

Received in revised form 1 March 1977. 
where $\zeta(n)$ has its set of a.s. limit points confined to $[-1,1]$ with $\lim \sup _{n \rightarrow \infty} \zeta(n)=+1$ a.s., $\lim \inf _{n \rightarrow \infty} \zeta(n)=-1$ a.s. It seems important to write these limit results in a form which highlights their role as rate results about the SLLN, rather than in the more familiar way which suppresses this relationship.

In this paper we shall be concerned with $\left\{\sum_{i=1}^{n} X_{i}, F_{n}, n \geqq 1\right\}$, a zero mean, square integrable martingale. Square integrability is needed for the most widely applicable rate results so it is assumed at the outset. Now suppose $\lim _{n \rightarrow \infty} E\left(\sum_{i=1}^{n} X_{i}\right)^{2}=\sum_{i=1}^{\infty} E X_{i}^{2}<\infty$. Then, the martingale convergence theorem gives $\sum_{i=1}^{n} X_{i} \stackrel{\text { a.s. }}{\longrightarrow} \sum_{i=1}^{\infty} X_{i}$, and in asking for rate results on this convergence we look for multipliers $\left\{B_{n}, n \geqq 1\right\}$, possibly random but increasing to infinity (a.s.), such that $B_{n} \sum_{i=n}^{\infty} X_{i}$ has behaviour of the type of the CLT or LIL. This will be the subject of Section 2. Of course these results involve a tail sum of martingale differences and the ordinary CLT and LIL for martingales do not bear directly on this context. Section 3 provides an example, involving the Pólya urn scheme.

\section{Central limit and iterated logarithm results}

For cases where the $X_{i}$ are not independent, the literature on this problem amounts to a variety of special results for particular contexts. For example, if $\left\{Z_{0}=1, Z_{1}, Z_{2}, \cdots\right\}$ denotes a supercritical Bienaymé-Galton-Watson process whose offspring distribution has mean $m(>1)$ and finite variance $\sigma^{2}$, then an application of the martingale convergence theorem gives $m^{-n} Z_{n} \stackrel{\text { a.s. }}{\longrightarrow} W$, where $W$ turns out to be a non-degenerate random variable. It is further known that, conditional on non-extinction,

$$
m^{n} Z_{n}^{-1 / 2}\left(m^{-n} Z_{n}-W\right) \stackrel{\mathrm{d}}{\rightarrow} N\left(0, \sigma^{2}\left(m^{2}-m\right)^{-1}\right) .
$$

This is a result of the type under discussion. It rests, however, on results for sums of independent and identically distributed random variables since $Z_{n}-m^{n} W$ can be represented as a sum of $Z_{n}$ independent random variables, each with the distribution of $1-W$ (which has mean zero and variance $\left.\sigma^{2}\left(m^{2}-m\right)^{-1}\right)$. Full details can be found in Chapter I of Athreya and Ney (1972).

For the case of independent $X_{i}$ there are some nice duality results in the literature. Chow and Teicher (1973) have obtained the following LIL's.

Proposition $A$. Let $\left\{X_{n}, n \geqq 1\right\}$ be independent and identically distributed random variables with $E X_{n}=0$ and $E X_{n}^{2}=1$ and $\left\{a_{n}, n \geqq 1\right\}$ be real constants.

(a) If $\sum_{1}^{n} a_{j}^{2} \rightarrow \infty$ as $n \rightarrow \infty$ and $\left(a_{n}^{2} / \sum_{1}^{n} a_{j}^{2}\right) \leqq C / n, n \geqq 1$, for some $C$ in $(0, \infty)$, then

$$
\underset{n \rightarrow \infty}{\limsup }\left(2 \sum_{j=1}^{n} a_{j}^{2} \log \log \sum_{j=1}^{n} a_{j}^{2}\right)^{-1 / 2} \sum_{j=1}^{n} a_{j} X_{j}=1 \text { a.s. }
$$

(b) If $\Sigma_{1}^{\infty} a_{j}^{2}<\infty$ and $\left(a_{n}^{2} / \Sigma_{n}^{\infty} a_{j}^{2}\right) \leqq C / n, n \geqq 1$, for some $C$ in $(0, \infty)$, then 


$$
\limsup _{n \rightarrow \infty}\left(2 \sum_{j=n}^{\infty} a_{j}^{2} \log \log \left(\sum_{j=n}^{\infty} a_{j}^{2}\right)^{-1}\right)^{-1 / 2} \sum_{j=n}^{\infty} a_{j} X_{j}=1 \text { a.s. }
$$

The proofs given by Chow and Teicher for these results are entirely classical, using truncations and exponential inequalities.

Much more important from the point of view of general theory is the paper of Barbour (1974). Barbour works with independent random variables but sketches a method which is widely applicable, well beyond this case. The method has its origins in work of Whitt (1972) in a rather different context. Barbour's basic result is the following duality form for the Lindeberg-Feller version of the CLT.

Proposition $B$. Let $\left\{X_{n}, n \geqq 1\right\}$ be independent random variables with zero means and finite variances.

(a) Suppose that $s_{n}^{2}=\sum_{k=1}^{n} E X_{k}^{2} \rightarrow \infty$ as $n \rightarrow \infty$. If

$$
s_{n}^{-2} E X_{n}^{2} \rightarrow 0 \text { as } n \rightarrow \infty,
$$

and

$$
S_{n}^{-2} \sum_{k=1}^{n} E\left(X_{k}^{2} I\left(\left|X_{k}\right|>\varepsilon s_{n}\right)\right) \rightarrow 0, \forall \varepsilon>0,
$$

then

$$
s_{n}^{-1} \sum_{k=1}^{n} X_{k} \stackrel{d}{\rightarrow} N(0,1)
$$

(b) Suppose that $s_{n}^{2}=\sum_{k=n}^{\infty} E X_{k}^{2} \rightarrow 0$ as $n \rightarrow \infty$. If

$$
s_{n}^{-2} E X_{n}^{2} \rightarrow 0 \text { as } n \rightarrow \infty,
$$

and

$$
S_{n}^{-2} \sum_{k=n}^{\infty} E\left(X_{k}^{2} I\left(\left|X_{k}\right|>\varepsilon s_{n}\right)\right) \rightarrow 0, \quad \forall \varepsilon>0,
$$

then

$$
s_{n}^{-1} \sum_{k=n}^{\infty} X_{k} \stackrel{\mathrm{d}}{\rightarrow} N(0,1)
$$

Barbour also purports to sketch a proof of a duality result for the Kolmogorov form of the LIL but this is unfounded as the necessary prerequisite of an ordinary functional LIL under the Kolmogorov condition has yet to be established. Nevertheless, useful basic methodology is provided and we shall take this up for the martingale case.

First a remark about notation. Let $J$ be an interval of the form $[0, L]$ for some $L<\infty$ or $[0, \infty)$. Let $D(J)$ be the space of right-continuous real-valued functions on $J$ with left limits. We shall understand that $D(J)$ is endowed with the Skorokhod $J_{1}$ topology in the absence of another prescription. 
The basic setting for Barbour's methodology is $T$, the subspace of $D[0, \infty)$ consisting of those functions $x$ which satisfy

$$
\begin{aligned}
& \underset{t \rightarrow \infty}{\limsup } t^{-1}|x(t)|=0, \\
& \int_{1}^{\infty} u^{-2}|x(u)| d u<\infty \\
& \int_{0}^{1} u^{-1}|x(u)| d u<\infty
\end{aligned}
$$

Let $m$ be the metric on $T$ such that $m(x, y)$ is the infimum of those $\varepsilon>0$ for which there exists some continuous strictly increasing function $\lambda:[0, \infty) \rightarrow[0, \infty)$ with $\lambda(0)=0$, such that

$$
\begin{gathered}
\sup _{t \geq 0}|x(t)-y(\lambda(t))| /(t \vee 1)<\varepsilon, \\
\int_{1}^{\infty} u^{-2}|x(u)-y(\lambda(u))| d u<\varepsilon, \\
\int_{0}^{1} u^{-1}|x(u)-y(\lambda(u))| d u<\varepsilon, \\
\sup _{t \neq s}\left|\log \frac{\lambda(t)-\lambda(s)}{t-s}\right|<\varepsilon .
\end{gathered}
$$

Let $T_{1}$ and $m_{1}$ be defined similarly but omitting the restrictions $C, C^{\prime}$. We shall use a star to denote the corresponding subspaces of $D^{*}[0, \infty)$, the space of left-continuous functions with right limits on $[0, \infty)$.

We consider the mapping $g: T \rightarrow T_{1}^{*}$ or: $T^{*} \rightarrow T_{1}$ defined by

$$
g(x)(0)=0 ; \quad g(x)(s)=-s x\left(s^{-1}\right)+\int_{s^{-1}}^{\infty} u^{-2} x(u) d u, \quad 0<s<\infty .
$$

That is, $g(x)(s)$ is the stochastic integral $\int_{s^{-1}}^{\infty} u^{-1} d x(u)$ defined by its integration by parts formula. It is easily checked that the mapping is continuous (e.g. as with Lemmas 2.1 and 3.7 of Whitt (1972)) and that for $x \in T \cup T^{*}$ with $x(0)=0$, $g(g(x))=x$. The mapping provides a device for reading results for tail sums off from those of ordinary sums (and vice versa) and we shall explore its ramifications for the case of martingale differences.

Recall that $\left\{\sum_{i=1}^{n} X_{i}, F_{n}, n \geqq 1\right\}$ is a zero mean, square integrable martingale with $\sum_{i=1}^{\infty} E X_{i}^{2}<\infty$. We set $S_{n}=\sum_{k=n}^{\infty} X_{k}$ and $s_{n}^{2}=E S_{n}^{2}=\sum_{k=n}^{\infty} E X_{k}^{2}$. We shall initially be dealing with the case where $s_{n}^{-2} \sum_{k=n}^{\infty} X_{k}^{2} \stackrel{\text { a.s. }}{\longrightarrow} \eta^{2}, \eta^{2}$ being some positive and a.s. finite random variable. 
Let $\left\{Z_{n}, n \geqq 1\right\}$ be a monotone sequence of positive random variables defined by $Z_{n}^{2}=\max _{k \leqq n} s_{k}^{-2}\left(E\left(\eta^{2} \mid F_{k-1}\right)\right)^{-1}$. Then $Z_{n}$ is $F_{n-1}$-measurable and $Z_{n}^{2} \sim s_{n}^{-2} \eta^{-2}$ a.s. $n \rightarrow \infty$ since $E\left(\eta^{2} \mid F_{n-1}\right) \stackrel{\text { a.s. }}{\longrightarrow} \eta^{2}$ as $n \rightarrow \infty$. Put

$$
Y_{n}(t)=Z_{n} f(n) S_{k(n, t)+1}, \quad t \geqq 0,
$$

where $f(n)$ is a normalization function and

$$
k(n, t)=\max \left[j \mid t Z_{j}^{2} \leqq Z_{n}^{2}\right] .
$$

Then, formally,

$$
g\left(Y_{n}\right)(s)=Z_{n}^{-1} f(n) \sum_{j=1}^{k\left(n, s^{-1}\right)} X_{j} Z_{j}^{2}, \quad s>0,
$$

and we shall apply standard martingale results to $g\left(Y_{n}\right)$.

At this stage we need to be specific about the conditions imposed. We shall establish the following theorem which, for the purposes of the exposition, is described in terms of a duality result.

Theorem 1. Let $\left\{\sum_{1}^{n} X_{i}, F_{n}, n \geqq 1\right\}$ be a zero mean square integrable martingale.

(a) Write $W_{n}^{2}=\sum_{1}^{n} X_{i}^{2}$ and $s_{n}^{2}=E W_{n}^{2}$. Suppose that $s_{n} \rightarrow \infty$ and

$$
s_{n}^{-2} \sum_{1}^{n} X_{i}^{2}=s_{n}^{-2} W_{n}^{2} \stackrel{\mathrm{p}}{\rightarrow} \eta^{2}
$$

where $\eta^{2}$ is some a.s. finite and non-zero random variable. If, in addition to (i) we have

$$
s_{n}^{-2} E\left(\max _{1 \leqq k \leqq n} X_{k}^{2}\right) \rightarrow 0 \quad \text { as } \quad n \rightarrow \infty,
$$

then

$$
W_{n}^{-1} \sum_{1}^{n} X_{i} \stackrel{\mathrm{d}}{\rightarrow} N(0,1) \quad \text { and } \quad s_{n}^{-1} \sum_{1}^{n} X_{i} \stackrel{\mathrm{d}}{\rightarrow} \eta^{\prime} N(0,1)
$$

where $\eta^{\prime}$ is independent of $N(0,1)$ and distributed as $\eta$.

On the other hand, if the convergence in (a)(i) is strengthened to a.s. convergence and in addition we have

$$
\sum_{1}^{\infty} s_{j}^{-1} E\left[\left|X_{j}\right| I\left(\left|X_{j}\right|>\varepsilon s_{j}\right)\right]<\infty, \quad \forall \varepsilon>0,
$$

and

$$
\sum_{1}^{\infty} s_{j}^{-4} E\left[X_{j}^{4} I\left(\left|X_{j}\right| \leqq \delta s_{j}\right)\right]<\infty \quad \text { for some } \delta>0,
$$

then, writing $\phi(t)=(2 \log \log (t \vee 3))^{1 / 2}$, 


$$
\left(W_{n} \phi\left(W_{n}\right)\right)^{-1} \sum_{j=1}^{n} X_{j}=\theta_{1}(n)
$$

has for its set of a.s. limit points the closed interval $[-1,1]$ with

$$
\limsup _{n \rightarrow \infty} \theta_{1}(n)=+1 \text { a.s., } \quad \liminf _{n \rightarrow \infty} \theta_{1}(n)=-1 \text { a.s. }
$$

(b) Write $W_{n}^{2}=\sum_{n}^{\infty} X_{i}^{2}$ and $s_{n}^{2}=E W_{n}^{2}$. Suppose that $s_{n} \rightarrow 0$ and

$$
s_{n}^{-2} \sum_{n}^{\infty} X_{j}^{2}=s_{n}^{-2} W_{n}^{2} \stackrel{\mathrm{p}}{\rightarrow} \eta^{2}
$$

where $\eta^{2}$ is some a.s. finite and non-zero random variable. If, in addition to (b)(i) we have

$$
s_{n}^{-2} E\left(\max _{k \geqq n} X_{k}^{2}\right) \rightarrow 0 \quad \text { as } \quad n \rightarrow \infty,
$$

then

$$
W_{n}^{-1} \sum_{n}^{\infty} X_{i} \stackrel{\mathrm{d}}{\rightarrow} N(0,1) \quad \text { and } \quad s_{n}^{-1} \sum_{n}^{\infty} X_{i} \stackrel{\mathrm{d}}{\rightarrow} \eta^{\prime} N(0,1)
$$

where $\eta^{\prime}$ is independent of $N(0,1)$ and distributed as $\eta$.

On the other hand, if the convergence in (b)(i) is strengthened to a.s. convergence and in addition we have

$$
\sum_{1}^{\infty} s_{j}^{-1} E\left[\left|X_{j}\right| I\left(\left|X_{j}\right|>\varepsilon s_{j}\right)\right]<\infty, \quad \forall \varepsilon>0,
$$

and

$$
\sum_{1}^{\infty} s_{j}^{-4} E\left[X_{j}^{4} I\left(\left|X_{j}\right| \leqq \delta s_{j}\right)\right]<\infty \quad \text { for some } \quad \delta>0,
$$

then

$$
\left(W_{n} \phi\left(W_{n}^{-1}\right)\right)^{-1} \sum_{j=n}^{\infty} X_{j}=\theta_{2}(n)
$$

has for its set of a.s. limit points the closed interval $[-1,1]$ with

$$
\limsup _{n \rightarrow \infty} \theta_{2}(n)=+1 \text { a.s., } \quad \liminf _{n \rightarrow \infty} \theta_{2}(n)=-1 \text { a.s. }
$$

The conditions given in the statement of this theorem are frequently not the most convenient for applications and some useful variants are given in the following corollaries.

Corollary 1. (a) Sufficient conditions for (a)(i) and (a)(ii) in Theorem 1 are 
(a)(i')

$$
s_{n}^{-2} \sum_{1}^{n} E\left(X_{i}^{2} \mid F_{i-1}\right) \stackrel{\mathrm{p}}{\rightarrow} \eta^{2}
$$

and

(a)(ii') $\quad s_{n}^{-2} \sum_{1}^{n} E\left(X_{i}^{2} I\left(\left|X_{i}\right|>\varepsilon s_{n}\right)\right) \rightarrow 0, \quad \forall \varepsilon>0$.

(b) Sufficient conditions for (b)(i) and (b)(ii) in Theorem 1 are

$$
s_{n}^{-2} \sum_{n}^{\infty} E\left(X_{i}^{2} \mid F_{i-1}\right) \stackrel{\mathrm{p}}{\rightarrow} \eta^{2}
$$

and

(b)(ii')

$$
s_{n}^{-2} \sum_{n}^{\infty} E\left(X_{i}^{2} I\left(\left|X_{i}\right|>\varepsilon s_{n}\right)\right) \rightarrow 0, \quad \forall \varepsilon>0 .
$$

Corollary 2. (a) $[(\mathrm{b})]$. Put $V_{n}^{2}=\sum_{1}^{n} E\left(X_{i}^{2} \mid F_{i-1}\right)\left[=\sum_{n}^{\infty} E\left(X_{i}^{2} \mid F_{i-1}\right)\right]$. A sufficient condition for the replacement of $W_{n}$ by $V_{n}$ in Part (a) [(b)] of Theorem 1 is that $\sum_{1}^{\infty} S_{n}^{-2}\left(X_{n}^{2}-E\left(X_{n}^{2} \mid F_{n-1}\right)\right)$ converges a.s.

As a preliminary to the proofs of these results we shall establish the following lemma.

Lemma 1 . Let $\left\{a_{k}, k \geqq 1\right\}$ and $\left\{b_{k}, k \geqq 1\right\}$ be sequences of real numbers with $a_{k} \geqq 0, \forall k$.

(i) If $\sum_{1}^{\infty} a_{k}<\infty$ and $\left(a_{k} / \sum_{j=k}^{\infty} a_{j}\right) \rightarrow l$, some $0 \leqq l<1$, as $k \rightarrow \infty$, then

$$
\left(\sum_{j=n}^{\infty} a_{j}\right) \sum_{k=1}^{n}\left[a_{k}\left(\sum_{j=k}^{\infty} a_{j}\right)^{-2}\right] \rightarrow 1 \text { as } n \rightarrow \infty .
$$

(ii) If $a_{n} \uparrow \infty$ and the series $\sum_{1}^{\infty} a_{k} b_{k}$ converges, then $\sum_{k=n}^{\infty} b_{k}=o\left(a_{n}^{-1}\right)$ as $n \rightarrow \infty$.

Proof. (i) Write $c_{n}=\left(\sum_{j=n}^{\infty} a_{j}\right)^{-1}$, so that what we have to show is

$$
c_{n}^{-1} \sum_{k=1}^{n} c_{k}^{2}\left(c_{k}^{-1}-c_{k+1}^{-1}\right) \rightarrow 1 \text {. }
$$

However, (6) can be rewritten as

$$
c_{n}^{-1} \sum_{k=1}^{n} c_{k} c_{k+1}^{-1}\left(c_{k+1}-c_{k}\right) \rightarrow 1=c_{n}^{-1} \sum_{k=1}^{n-1}\left(c_{k+1}-c_{k}\right)+c_{n}^{-1} c_{1}
$$

or equivalently

$$
\left(1-c_{n} c_{n+1}^{-1}\right)+c_{n}^{-1} \sum_{k=1}^{n-1}\left(c_{k} c_{k+1}^{-1}-1\right)\left(c_{k+1}-c_{k}\right) \rightarrow 0 .
$$

The result (7) follows via the Toeplitz lemma since $1-c_{k} c_{k+1}^{-1} \rightarrow l$ as $k \rightarrow \infty$.

(ii) Let $K_{n}=\sup _{N \geqq M \geqq n}\left|\sum_{k=n}^{M} a_{k} b_{k}\right|$, so that $K_{n} \rightarrow 0$ as $n \rightarrow \infty$. 
From Abel's lemma,

$$
\left|\sum_{k=n}^{N} b_{k}\right| \leqq K_{n} / a_{n}
$$

and the required result follows upon letting $N \rightarrow \infty$.

Proof of Theorem 1. The results of Part (a) of the theorem are known and are just stated for the purpose of exhibiting the duality. The central limit result follows, for example, from Corollaries 2.1.1 and 2.2.1 of Hall (1977). The iterated logarithm result is essentially that of Hall and Heyde (1976). We shall thus confine our attention to the proof of Part (b) of the theorem.

We begin by using invariance principle versions of Part (a) and the methodology proposed by Barbour. For this purpose we assume a.s. convergence in (b)(i). This restriction will later be removed for the central limit case via other methods.

The first thing we need to check is that $g\left(Y_{n}\right)$ as given by (3) belongs to $T$ under the conditions of (b). As a prelude to this we observe that $s_{n+1}^{2} s_{n}^{-2} \rightarrow 1$ as $n \rightarrow \infty$. This is easily deduced from (b)(ii) for the central limit case. For the iterated logarithm case, on the other hand, we have for any $\varepsilon>0$,

$$
S_{n}^{-1}\left|X_{n}\right| \leqq \varepsilon+S_{n}^{-1}\left|X_{n}\right| I\left(\left|X_{n}\right|>\varepsilon s_{n}\right) \stackrel{\text { a.s. }}{\longrightarrow} \varepsilon
$$

using (b)(iii) so that $s_{n}^{-2} X_{n}^{2} \stackrel{\text { a.s. }}{\longrightarrow} 0$ and the desired result follows readily using (b)(i).

Next, note that

$$
\frac{X_{n}^{2}}{W_{n}^{2}}=\frac{W_{n}^{2}-W_{n+1}^{2}}{W_{n}^{2}}=1-\frac{S_{n+1}^{-2} W_{n+1}^{2}}{S_{n}^{-2} W_{n}^{2}} \frac{S_{n+1}^{2}}{S_{n}^{2}} \stackrel{\text { a.s. }}{\longrightarrow} 0
$$

and hence, using (b)(i), Lemma 1 (i) and $Z_{n}^{2} \sim W_{n}^{-2}$ a.s.,

$$
Z_{n}^{-2} \sum_{1}^{n} X_{k}^{2} Z_{k}^{4} \stackrel{\text { a.s. }}{\longrightarrow} 1 \text {. }
$$

With the aid of (8) and recalling that $Z_{k\left(n, s^{-1}\right)}^{2} \sim s Z_{n}^{2}$ as $s \rightarrow \infty$ we can deduce that

$$
s^{-3 / 4} g\left(Y_{n}\right)(s) \stackrel{\text { a.s. }}{\longrightarrow} 0 \text { as } s \rightarrow \infty .
$$

Indeed, in view of (8) the result (9) holds if

$$
\sum_{j=1}^{k\left(n, s^{-1}\right)} X_{j} Z_{j}^{2} /\left(\sum_{j=1}^{k\left(n, s^{-1}\right)} X_{j}^{2} Z_{j}^{4}\right)^{3 / 4} \stackrel{\text { a.s. }}{\longrightarrow} 0 \quad \text { as } \quad s \rightarrow \infty .
$$

However, (10) follows, using the Kronecker lemma, if

$$
\sum_{1}^{\infty}\left[X_{n} Z_{n}^{2} /\left(\sum_{1}^{n} X_{j}^{2} Z_{j}^{4}\right)^{3 / 4}\right] \text { converges a.s. }
$$


and hence if, using (8) again,

$$
\sum_{1}^{\infty} X_{n} s_{n}^{-1 / 2} \quad \text { converges a.s. }
$$

Then, the martingale convergence theorem gives (11) provided that

$$
\sum_{1}^{\infty} s_{n}^{-1} E X_{n}^{2}<\infty
$$

as is easily checked since $s_{n} \geqq s_{n+1}$ and

$$
\begin{aligned}
\sum_{1}^{\infty} s_{n}^{-1}\left(s_{n}^{2}-s_{n+1}^{2}\right) & =\sum_{1}^{\infty} s_{n}^{-1}\left(s_{n}+s_{n+1}\right)\left(s_{n}-s_{n+1}\right) \leqq 2 \sum_{1}^{\infty}\left(s_{n}-s_{n+1}\right) \\
& =2 s_{1}<\infty
\end{aligned}
$$

and the proof of (9) is complete.

The prerequisite conditions for $g\left(Y_{n}\right)$ to belong to $T$ are now easily verified. The relation (9) ensures that (A) and (B) hold, while (C) is satisfied as the defining integral reduces to a finite sum.

We now take $f(n)=1$ and focus attention on the central limit part of (b). We begin by applying a minor modification of Corollary 2.1.1 of Hall (1977) to the martingale array $\left\{s_{n} \sum_{j=1}^{k} X_{j} Z_{j}^{2}, F_{k}, 1 \leqq k \leqq n, n \geqq 1\right\}$. For $z \geqq 0$ let

$$
\xi_{n}(z)=Z_{n}^{-1} \sum_{j=1}^{k\left(n, z^{-1}\right)} X_{j} Z_{j}^{2}
$$

This formulation is not quite that of Hall but the modifications to the summation can be simply effected as in the proof of his Theorem 3.1. We take, in the notation of Hall's result, $F_{n j}=F_{j}$. In view of (8), we need only check that $s_{n}^{2} \max _{1 \leqq j \leqq n} X_{j}^{2} Z_{j}^{4} \stackrel{\mathrm{P}}{\rightarrow} 0$ and hence that $s_{n}^{2} \max _{1 \leqq j \leqq n} X_{j}^{2} s_{j}^{-4} \stackrel{\mathrm{p}}{\rightarrow} 0$. Furthermore, since $s_{n} \downarrow 0$ and $s_{n+1} s_{n}^{-1} \rightarrow 1$ we can, given $\varepsilon>0$, choose a sequence $\{j(n)\}$ such that $s_{n}^{2} / s_{j(n)}^{2} \rightarrow \varepsilon^{\prime} \leqq \varepsilon$ as $n \rightarrow \infty$. Then,

$$
\begin{aligned}
0 & \leqq \limsup _{n \rightarrow \infty} s_{n}^{2} E\left(\max _{1 \leqq j \leqq n} X_{j}^{2} s_{j}^{-4}\right) \\
& \leqq \limsup _{n \rightarrow \infty} s_{n}^{2} E\left(\max _{1 \leqq j<j(n)} X_{j}^{2} s_{j}^{-4}\right)+\limsup _{n \rightarrow \infty} s_{n}^{2} E\left(\max _{j(n) \leqq j \leqq n} X_{j}^{2} s_{j}^{-4}\right) \\
& \leqq \limsup _{n \rightarrow \infty} s_{n}^{2} \sum_{j=1}^{j(n)-1}\left(s_{j}^{2}-s_{j+1}^{2}\right) s_{j}^{-4}+\limsup _{n \rightarrow \infty} s_{n}^{-2} E\left(\max _{j \geqq j(n)} X_{j}^{2}\right) \leqq \varepsilon^{\prime}
\end{aligned}
$$

in view of (b)(ii) and

$$
\sum_{j=1}^{j(n)-1}\left(s_{j}^{2}-s_{j+1}^{2}\right) s_{j}^{-4} \leqq \sum_{j=1}^{j(n)-1}\left(s_{j}^{2}-s_{j+1}^{2}\right) s_{j}^{-2} s_{j+1}^{-2}=\sum_{j=1}^{j(n)-1}\left(s_{j+1}^{-2}-s_{j}^{-2}\right)=s_{j(n)}^{-2} .
$$


The corollary in question then gives

$$
\left(\xi_{n}, s_{n}^{2} Z_{n}^{2}\right) \stackrel{\mathrm{d}}{\rightarrow}\left(W,\left(\eta^{\prime}\right)^{-2}\right)
$$

where $W$ is standard Brownian motion and $\eta^{\prime}$ has the same distribution as $\eta$ and is independent of $W$. The basic setting is $D[0,1]$ and, defining $\eta_{n}(z)=$ $s_{n}^{-1} Z_{n}^{-1} \xi_{n}(z)$, we have

$$
\xi_{n} \stackrel{\mathrm{d}}{\rightarrow} W \quad \text { and } \quad \eta_{n} \stackrel{\mathrm{d}}{\rightarrow} \eta^{\prime} W
$$

in this setting. However, the extension of the results (12) to $D[0, T], 0<T<\infty$, and indeed to $D[0, \infty)$ is entirely routine (e.g. see McLeish (1974), pp. 625-626).

Of course $\xi_{n}=g\left(Y_{n}\right)$ and $\eta_{n}$ is also an element of $T$ so it remains to check that the results (12) continue to apply in $(T, m)$. This however follows from the argument of Müller ((1968), p. 176, 177; see also Whitt (1972)) since the Kolmogorov inequality continues to hold for martingales. Then, using the continuous mapping theorem (e.g. Billingsley (1968), Theorem 5.1),

$$
g\left(\xi_{n}\right)=g\left(g\left(Y_{n}\right)\right)=Y_{n} \stackrel{\mathrm{d}}{\rightarrow} g(W) \stackrel{\mathrm{d}}{=} W
$$

while

$$
g\left(\eta_{n}\right)=s_{n}^{-1} Z_{n}^{-1} g\left(g\left(Y_{n}\right)\right)=s_{n}^{-1} Z_{n}^{-1} Y_{n} \stackrel{\mathrm{d}}{\rightarrow} g\left(\eta^{\prime} W\right) \stackrel{\mathrm{d}}{=} \eta^{\prime} W
$$

and the results (5) follow immediately.

It should be noted that the functional limit results just obtained offer scope for a variety of other important results via the continuous mapping theorem. From the convergence $Y_{n} \stackrel{\mathrm{d}}{\rightarrow} W$ in $T_{1}^{*}$ we can extract $Y_{n} \stackrel{\mathrm{d}}{\rightarrow} W$ in $\left(D^{*}[0,1], d_{0}\right)$ and hence in $\left(D^{*}[0,1], d\right)$, and $d_{0}$ being the metrics discussed by Billingsley ((1968), pp. 111-113) which generate the Skorokhod topology. Then, for example, the mapping $h: D^{*}[0,1] \rightarrow R$ defined by $h(x)(t)=\sup _{0 \leqq t \leqq 1}|x(t)|$ is continuous and the continuous mapping theorem gives $h\left(Y_{n}\right) \stackrel{d}{\rightarrow} h(W)$, or equivalently,

$$
W_{n}^{-1} \sup _{k \geqq n}\left|\sum_{j=k}^{\infty} X_{j}\right| \stackrel{\mathrm{d}}{\rightarrow} \sup _{0 \leqq t \leqq 1}|W(t)| .
$$

Next we take $f(n)=\left(2 \log \log \left(s_{n}^{-1} \vee 3\right)\right)^{-1 / 2}$ in (1) and focus attention on the iterated logarithm part of (b). It is our object to show that $\left\{g\left(Y_{n}\right), n \geqq 1\right\}$ has compact closure a.s. with set of limit points $K, K$ being the set of absolutely continuous real functions on $[0, \infty)$ for which $x(0)=0$ and $\int_{0}^{\infty}[\dot{x}(t)]^{2} d t \leqq 1, \dot{x}$ denoting the derivative of $x$ determined almost everywhere with respect to Lebesgue measure. The iterated logarithm part of (b) is then easily extracted 
from the result that $\left\{Y_{n}=g\left(g\left(Y_{n}\right)\right), n \geqq 1\right\}$ has compact closure a.s. with set of limit points $g(K)=K$, while $Z_{n} W_{n} \phi\left(W_{n}^{-1}\right)\left[\phi\left(Z_{n}^{-1}\right)\right]^{-1} \stackrel{\text { a.s. }}{\longrightarrow} 1$. That $K$ belongs to $T$ and $T^{*}$ follows readily from

$$
|x(t)|=\left|\int_{0}^{t} \dot{x}(u) d u\right| \leqq\left\{\int_{0}^{t} d u \int_{0}^{t}[\dot{x}(u)]^{2} d u\right\}^{1 / 2} \leqq t^{1 / 2} .
$$

We shall apply Theorem 1 of Hall and Heyde (1976) which deals with the setting $C[0,1]$ but whose scope can be extended to cover the problem in hand. The martingale to which the theorem is applied is $\left\{\sum_{j=1}^{n} X_{j} Z_{j}^{2}, F_{n}, n \geqq 1\right\}$ and for the $Z_{j}$ and $W_{j}$ of the theorem we use $\delta s_{j} Z_{j}^{2}$ and $Z_{j}$ respectively. The checking of the conditions is routine and will not be exhibited. It follows quite closely upon the proof of Corollary 2 of Hall and Heyde (1976) and makes repeated use of (8) and the $F_{j-1}$-measurability of $Z_{j}$. Furthermore, the extension of this setting from $C[0,1]$ to $C[0, \infty)$ is a standard exercise. For example, the argument provided in the proof of Theorem 1.5.15 of Vervaat (1972) can be employed. The topology for $C[0, \infty)$ is that of uniform convergence on compacta. Then, writing for $0 \leqq u<\infty, k=k\left(n, u^{-1}\right)$ as given by (2) and

$$
\mu_{n}(u)=\left[Z_{n} \phi\left(Z_{n}\right)\right]^{-1}\left[\sum_{j=1}^{k} X_{j} Z_{j}^{2}+\left(u Z_{n}^{2}-Z_{k}^{2}\right)\left(Z_{k+1}^{2}-Z_{k}^{2}\right)^{-1} X_{k+1} Z_{k+1}^{2}\right],
$$

we have that $\left\{\mu_{n}, n \geqq 1\right\}$ is relatively compact in $C[0, \infty)$ and the set of its a.s. limit points coincides with $K$.

Next, we move to $D[0, \infty)$ and note that the Skorokhod $J_{1}$ topology relativized to $C[0, \infty)$ coincides there with the topology of uniform convergence on compacta. We can use the metric

$$
\psi(x, y)=\int_{0}^{\infty} \min \left\{1, \rho_{[0, t]}\left(\left.x\right|_{[0, t]},\left.Y\right|_{[0, t]}\right\} e^{-t} d t\right.
$$

for $x, y \in D[0, \infty)$ where $\rho_{[0, t]}$ is a metric on $D[0, t]$ which generates the $J_{1}$ topology on $D[0, t]$ and $\left.z\right|_{[0, t]}$ denotes the restriction of $z$ to $[0, t]$ (Vervaat (1972), p. 23).

In $D[0, \infty)$ we set

$$
\nu_{n}(u)=\left[Z_{n} \phi\left(Z_{n}\right)\right]^{-1} \sum_{j=1}^{k} X_{j} Z_{j}^{2}, \quad 0 \leqq u<\infty .
$$

Then, $\psi\left(\nu_{n}, \mu_{n}\right) \stackrel{\text { a.s. }}{\longrightarrow} 0$ as $n \rightarrow \infty$ provided that

$$
\int_{0}^{\infty} \min \left\{1, \sup _{0 \leqq u \leqq t}\left|\nu_{n}(u)-\mu_{n}(u)\right|\right\} e^{-t} d t \stackrel{\text { a.s. }}{\longrightarrow} 0 \text { as } n \rightarrow \infty,
$$

by choosing a version of $\rho_{[0, t]}$ which is dominated by the uniform metric. Further, (13) holds if 


$$
\int_{0}^{\infty} \min \left\{1,\left[Z_{n} \phi\left(Z_{n}\right)\right]^{-1} \sup _{0 \leqq u \leqq t}\left|X_{k+1}\right| Z_{k+1}^{2}\right\} e^{-t} d t \stackrel{\text { a.s. }}{\longrightarrow} 0 \text { as } n \rightarrow \infty
$$

which is easily verified since, using (8),

$$
\left[Z_{n} \phi\left(Z_{n}\right)\right]^{-2} \sup _{0 \leqq u \leqq t} X_{k+1}^{2} Z_{k+1}^{4}=O\left(Z_{n}^{-2} \sum_{j=1}^{k\left(n, t^{-1)+1}\right.} X_{j}^{2} Z_{j}^{4}\right)=o(t) \text { a.s. }
$$

as $t \rightarrow \infty$. It then follows that $\left\{\nu_{n}, n \geqq 1\right\}$ is relatively compact in $(D[0, \infty), \psi)$ and the set of its a.s. limit points coincides with $K$.

Finally, we move to $(T, m)$. The Borel $\sigma$-field associated with $(T, m)$ coincides with the $\sigma$-field on $T$ generated by the coordinate projections. This can be verified using obvious modifications of the arguments on pp. 121-122 of Billingsley (1968) (see also Lemma 3.5 of Whitt (1972)). Further, similar arguments show that the Borel $\sigma$-field associated with $D[0, \infty)$ for the Skorokhod topology is that generated by the coordinate projections. Thus, relative compactness of $\left\{\nu_{n}, n \geqq 1\right\}$ in $\{D[0, \infty), \psi\}$ implies the same property in $(T, m)$. Since $K \subset T$ and $f(n) \sim\left[\phi\left(Z_{n}\right)\right]^{-1}$ a.s. as $n \rightarrow \infty$, the proof of the iterated logarithm part of (b) is complete.

In the previous work we have dealt with the case of a.s. convergence in (b)(i); we now weaken this to convergence in probability for the purposes of proving the relevant central limit results.

We choose a monotone sequence $\left\{k_{n}, n \geqq 1\right\}$ of positive integers increasing so fast that $s_{n}^{-2} s_{n+k_{n}+1}^{2} \rightarrow 0$ as $n \rightarrow \infty$. Further, we introduce the array of random variables $\left\{X_{n j}, j \geqq 1, n \geqq 1\right\}$ defined by $X_{n j}=s_{n}^{-1} X_{n+j}$. Then, from (b)(i),

$$
\sum_{j=1}^{k_{n}} X_{n j}^{2}=s_{n}^{-2} \sum_{j=n}^{n+k_{n}} X_{j}^{2} \stackrel{\text { p }}{\rightarrow} \eta^{2}
$$

since $s_{n}^{-2} \sum_{j=n+k_{n}+1}^{\infty} X_{j}^{2 L_{1}} \rightarrow 0$ in view of the choice of $\left\{k_{n}\right\}$. Also, using (b)(ii),

$$
E\left(\max _{j \leqq k_{n}} X_{n j}^{2}\right)=s_{n}^{-2} E\left(\max _{n \leqq j \leqq n+k_{n}} X_{j}^{2}\right) \leqq s_{n}^{-2} E\left(\max _{j \geqq n} X_{j}^{2}\right) \rightarrow 0 .
$$

The $\sigma$-fields $F_{n j}=F_{n+j}$ satisfy the nesting condition $F_{n j} \subseteq F_{n+1, j}$ so the conditions of Corollaries 2.1.1 and 2.2.1 of Hall (1977) are satisfied and

$$
\begin{gathered}
\sum_{j=1}^{k_{n}} X_{n j}=s_{n}^{-1} \sum_{j=n}^{n+k_{n}} X_{j} \stackrel{\mathrm{d}}{\rightarrow} \eta^{\prime} N(0,1) \\
\left\{\sum_{j=1}^{k_{n}} X_{n j}^{2}\right\}^{-1 / 2} \sum_{j=1}^{k_{n}} X_{n j}=\left\{\sum_{j=n}^{n+k_{n}} X_{j}^{2}\right\}^{-1 / 2} \sum_{j=n}^{n+k_{n}} X_{j} \stackrel{\mathrm{d}}{\rightarrow} N(0,1) .
\end{gathered}
$$

The desired results (4) are then immediate since $s_{n}^{-2} \sum_{j=n+k_{n}+1}^{\infty} X_{j}^{2 L_{1}} \rightarrow 0$. This completes the proof of the theorem.

It is interesting to note that a minor reformulation of results of Hall (1977) 
provides a convenient general result from which the central limit results of both Parts (a) and (b) of Theorem 1 can be derived. This is given in the following theorem.

Theorem 2. Let $\left\{X_{n j}, F_{n j}, j \geqq 1\right\}$ denote a martingale difference sequence for each $n \geqq 1$ which is subject to the condition

$$
\sum_{j=1}^{\infty} E\left(X_{n j}^{2}\right)<\infty, \quad \forall n .
$$

If $F_{n j} \subseteq F_{n+1, j}$ for all $j \geqq 1$ and in addition

$$
\sum_{1}^{\infty} X_{n j}^{2} \stackrel{p}{\rightarrow} \eta^{2}(>0 \text { a.s. })
$$

and

$$
E\left(\max _{j} X_{n j}^{2}\right) \rightarrow 0
$$

as $n \rightarrow \infty$, then

$$
\sum_{1}^{\infty} X_{n j} \stackrel{\mathrm{d}}{\rightarrow} \eta^{\prime} N(0,1) \text { and }\left(\sum_{1}^{\infty} X_{n j}^{2}\right)^{-1 / 2} \sum_{1}^{\infty} X_{n j} \stackrel{\mathrm{d}}{\rightarrow} N(0,1),
$$

$\eta^{\prime}$ being independent of $N(0,1)$ and distributed as $\eta$.

These results follow from Corollaries 2.1.1 and 2.2.1 of Hall (1977) in similar fashion to those just discussed in the last part of the proof of Theorem 1. The virtue of this formulation was pointed out to the author by P. G. Hall. The central limit results of Part (a) of Theorem 1 can then be extracted by taking $X_{n j}=\left(\sum_{k=1}^{n} E X_{k}^{2}\right)^{-1 / 2} X_{j}, 1 \leqq j \leqq n ; X_{n j}=0, j>n$, and $F_{n j}=F_{j}$. Those of Part (b) follow by taking $X_{n j}=\left(\sum_{k=n}^{\infty} E X_{k}^{2}\right)^{-1 / 2} X_{n+j}, F_{n j}=F_{n+j}, j \geqq 1, n \geqq 1$, as in the above proof.

Proof of Corollary 1. Here we shall confine attention to the proof of Part (b). That for Part (a) is entirely analogous.

Take arbitrary $\delta>0$. Then,

$$
\begin{aligned}
s_{n}^{-2} E\left(\max _{k \geqq n} X_{k}^{2}\right) & \leqq s_{n}^{-2} E\left(\max _{k \geqq n} X_{k}^{2} I\left(\left|X_{k}\right| \leqq \delta s_{n}\right)\right)+s_{n}^{-2} E\left(\max _{k \geqq n} X_{k}^{2} I\left(\left|X_{k}\right|>\delta s_{n}\right)\right) \\
& \leqq \delta^{2}+s_{n}^{-2} \sum_{k=n}^{\infty} E\left(X_{k}^{2} I\left(\left|X_{k}\right|>\delta s_{n}\right)\right) \rightarrow \delta^{2} \text { as } n \rightarrow \infty
\end{aligned}
$$

which gives (b)(ii). To obtain (b)(i) we first note that (b)(ii') ensures

$$
s_{n}^{-2} \sum_{k=n}^{\infty}\left[X_{k}^{2} I\left(\left|X_{k}\right|>\varepsilon s_{n}\right)-E\left(X_{k}^{2} I\left(\left|X_{k}\right|>\varepsilon s_{n}\right) \mid F_{k-1}\right)\right] \stackrel{L_{1}}{\rightarrow} 0,
$$

while 


$$
S_{n}^{-2} \sum_{k=n}^{\infty}\left[X_{k}^{2} I\left(\left|X_{k}\right| \leqq \varepsilon S_{n}\right)-E\left(X_{k}^{2} I\left(\left|X_{k}\right| \leqq \varepsilon S_{n}\right) \mid F_{k-1}\right)\right] \stackrel{L_{2}}{\rightarrow} 0
$$

provided that

$$
s_{n}^{-4} \sum_{k=n}^{\infty} E\left[X_{k}^{4} I\left(\left|X_{k}\right| \leqq \varepsilon s_{n}\right)\right] \rightarrow 0 .
$$

That this last result holds, however, is easily verified since for arbitrary $\delta$, $\varepsilon>\delta>0$,

$$
\begin{aligned}
s_{n}^{-4} \sum_{k=n}^{\infty} E\left[X_{k}^{4} I\left(\left|X_{k}\right| \leqq \varepsilon s_{n}\right)\right]= & s_{n}^{-4} \sum_{k=n}^{\infty} E\left[X_{k}^{4} I\left(\left|X_{k}\right| \leqq \delta s_{n}\right)\right] \\
& +s_{n}^{-4} \sum_{k=n}^{\infty} E\left[X_{k}^{4} I\left(\delta s_{n}<\left|X_{k}\right| \leqq \varepsilon s_{n}\right)\right] \\
\leqq & \delta^{2}+\varepsilon^{2} S_{n}^{-2} \sum_{k=n}^{\infty} E\left[X_{k}^{2} I\left(\left|X_{k}\right|>\delta s_{n}\right)\right] \rightarrow \delta^{2}
\end{aligned}
$$

as $n \rightarrow \infty$. The result (b)(i) then follows from (20) and (21) together with (b)(i').

It can be further shown, and is worth noting, that the pair of conditions (a)(i'), (a)(ii') [(b)(i'), (b)(ii')] is equivalent to the pair (a)(i), (a)(ii) [(b)(i), (b)(ii)] if $E \eta^{2}=1$. We omit the details.

Proof of Corollary 2. Part (a) is an immediate consequence of the Kronecker lemma and Part (b) of Lemma 1 (ii).

Next we show that Propositions $\mathrm{A}$ and $\mathrm{B}$ follow as a consequence of Theorem 1.

Proof of Proposition A. We use the martingale $\left\{\sum_{j=1}^{n} a_{j} X_{j}, n \geqq 1\right\}$.

First take the assumptions of Part (a) of Proposition A. To check the a.s. convergence form of (a)(i) of Theorem 1 we observe that it suffices to show $\left(\sum_{1}^{n} a_{j}^{2}\right)^{-1} \sum_{1}^{n} a_{j}^{2}\left(X_{j}^{2}-1\right) \stackrel{\text { a.s. }}{\longrightarrow} 0$, and this follows readily from Theorem 2 of Heyde (1968) since $\left(a_{n}^{2} / \sum_{1}^{n} a_{j}^{2}\right) \leqq C / n, n \geqq 1$.

To check (a)(iii), we note that

$$
\begin{aligned}
& \sum_{1}^{\infty}\left(\sum_{1}^{n} a_{j}^{2}\right)^{-1 / 2} E\left\{\left|a_{j} X_{j}\right| I\left(\left|X_{j}\right|>\varepsilon\left(\sum_{1}^{n} a_{j}^{2}\right)^{1 / 2}\right)\right\} \\
& \leqq C^{1 / 2} \sum_{1}^{\infty} n^{-1 / 2} E\left\{\left|X_{1}\right| I\left(\left|X_{1}\right|>\varepsilon C^{-1 / 2} n^{1 / 2}\right)\right\} \\
& \quad=C^{1 / 2} E\left\{\left|X_{1}\right| \sum_{1}^{\infty} n^{-1 / 2} I\left(\left|X_{1}\right|>\varepsilon C^{-1 / 2} n^{1 / 2}\right)\right\} \\
& \quad \leqq C^{1 / 2} E\left\{\left|X_{1}\right| \sum_{1}^{\left[C_{-}^{-2} X_{1}^{2}\right]} n^{-1 / 2}\right\}
\end{aligned}
$$


where $[x]$ denotes the integer part of $x$

$$
\begin{aligned}
& \leqq C^{1 / 2} E\left\{\left|X_{1}\right| \int_{0}^{C^{-2} X_{1}^{2}} x^{-1 / 2} d x\right\} . \\
& \leqq 2 C \varepsilon^{-1} E X_{1}^{2}<\infty .
\end{aligned}
$$

Finally, to check (a)(iv) we write $u_{n}=\sum_{1}^{n} a_{k}^{2} / a_{n}^{2}$ so that the required result holds if $\sum_{1}^{\infty} u_{n}^{-2} E\left\{X_{1}^{4} I\left(n^{1 / 2}<\left|X_{1}\right| \leqq \delta u_{n}^{1 / 2}\right)\right\}<\infty$ and $\Sigma_{1}^{\infty} u_{n}^{-2} E\left\{X_{1}^{4} I\left(\left|X_{1}\right| \leqq n^{1 / 2}\right)\right\}<$ $\infty$. However, the former holds since

$$
\begin{aligned}
\sum_{1}^{\infty} u_{n}^{-2} E\left\{X_{1}^{4} I\left(n^{1 / 2}<\left|X_{1}\right| \leqq \delta u_{n}^{1 / 2}\right)\right\} & \leqq \delta^{4} \sum_{1}^{\infty} P\left(\left|X_{1}\right|>n^{1 / 2}\right) \\
& =\delta^{4} E \sum_{1}^{\infty} I\left(\left|X_{1}\right|>n^{1 / 2}\right) \leqq \delta^{4} E X_{1}^{2}<\infty,
\end{aligned}
$$

and the latter since

$$
\begin{aligned}
\sum_{1}^{\infty} & u_{n}^{-2} E\left\{X_{1}^{4} I\left(\left|X_{1}\right| \leqq n^{1 / 2}\right)\right\} \\
& \leqq C^{2} \sum_{1}^{\infty} n^{-2} E\left\{X_{1}^{4} I\left(\left|X_{1}\right| \leqq n^{1 / 2}\right)\right\} \\
& \leqq C^{2} \sum_{n=1}^{\infty} n^{-2} \sum_{k=1}^{n} k E\left\{X_{1}^{2} I\left((k-1)^{1 / 2}<\left|X_{1}\right| \leqq k^{1 / 2}\right)\right\} \\
& =C^{2} \sum_{k=1}^{\infty} k E\left\{X_{1}^{2} I\left((k-1)^{1 / 2}<\left|X_{1}\right| \leqq k^{1 / 2}\right)\right\} \sum_{n=k}^{\infty} n^{-2} \\
& \leqq C^{2} E X_{1}^{2}<\infty .
\end{aligned}
$$

This completes the proof of Part (a) of Proposition A, the random norming being replaced by constants in view of (a)(i).

To prove Part (b) of Proposition A we begin by checking the a.s. convergence form of (b)(i) or equivalently, $\sum_{n}^{\infty} a_{j}^{2}\left(X_{j}^{2}-1\right) / \Sigma_{n}^{\infty} a_{j}^{2} \stackrel{\text { a.s. }}{\longrightarrow} 0$ as $n \rightarrow \infty$. Write $u_{n}=$ $\sum_{n}^{\infty} a_{j}^{2} / a_{n}^{2}$ and set

$$
\begin{aligned}
& W_{j}=X_{j}^{2}-1 \quad \text { if } \quad\left|X_{j}^{2}-1\right| \leqq u_{j} \\
& =0 \quad \text { otherwise. }
\end{aligned}
$$

Then, $P\left(W_{n} \neq X_{n}^{2}-1\right.$ i.o. $)=0$ since

$$
\begin{aligned}
\sum_{1}^{\infty} P\left(W_{n} \neq X_{n}^{2}-1\right)=\sum_{1}^{\infty} P\left(\left|X_{1}^{2}-1\right|>u_{n}\right) & \leqq \sum_{1}^{\infty} P\left(C\left|X_{1}^{2}-1\right|>n\right) \\
& \leqq E \sum_{1}^{\infty} I\left(C\left|X_{1}^{2}-1\right|>n\right) \\
& \leqq C E\left|X_{1}^{2}-1\right|<\infty
\end{aligned}
$$


and hence $\sum_{n}^{\infty} a_{j}^{2}\left(X_{j}^{2}-1\right) / \sum_{n}^{\infty} a_{j}^{2} \stackrel{\text { a.s. }}{\longrightarrow} 0$ if $\sum_{n}^{\infty} a_{j}^{2} W_{j} / \sum_{n}^{\infty} a_{j}^{2} \stackrel{\text { a.s. }}{\longrightarrow} 0$. Also, $E W_{n} \rightarrow 0$ as $n \rightarrow \infty$ and hence $\sum_{n}^{\infty} a_{j}^{2} E W_{j} / \sum_{n}^{\infty} a_{j}^{2} \rightarrow 0$ so it remains to prove $\sum_{n}^{\infty} a_{j}^{2}\left(W_{j}-E W_{j}\right) / \sum_{n}^{\infty} a_{j}^{2} \stackrel{\text { a.s. }}{\longrightarrow} 0$ which holds, by virtue of Part (ii) of Lemma 1, if $\sum_{1}^{\infty} u_{n}^{-1}\left(W_{n}-E W_{n}\right)$ converges a.s. However, the Kolmogorov convergence criterion gives the a.s. convergence of $\sum_{1}^{\infty} u_{n}^{-1}\left(W_{n}-E W_{n}\right)$ provided that $\sum_{1}^{\infty} u_{n}^{-2} E W_{n}^{2}<\infty$ and this last result holds since

$$
\sum_{1}^{\infty} u_{n}^{-2} E W_{n}^{2}=\sum_{1}^{\infty} u_{n}^{-2} E\left\{\left(X_{1}^{2}-1\right)^{2} I\left(\left|X_{1}^{2}-1\right| \leqq u_{n}\right)\right\}<\infty
$$

using the same argument as for the checking of (a)(iv) above. The remainder of the proof of Part (b) involving checking of the series Conditions (b)(iii) and (b)(iv) of Theorem 1 completely parallels the corresponding work for (a)(iii) and (a)(iv) above and is omitted.

Proof of Proposition $B$. The results are immediate from Corollary 1 and Theorem 1 . In this case we also have $\eta=1$ a.s.

\section{An example}

In order to give some idea of the scope of the results of the paper and the relevance of the conditions therein we shall give an example dealing with the well-known Pólya urn scheme.

Suppose we have an urn initially containing $b$ black and $r$ red balls. At each draw we remove a ball at random and then replace it, together with $c$ balls of the colour drawn. Let $b_{n}, r_{n}$ denote, respectively, the numbers of black and red balls in the urn after the $n$th drawing and write $Y_{n}=b_{n} /\left(b_{n}+r_{n}\right), n \geqq 0$, the proportion of black balls. Note also that $b_{n}+r_{n}=b+r+n c$.

Now it is well known that $\left\{Y_{n}, n \geqq 0\right\}$ is a martingale with respect to the past history $\sigma$-fields, that the martingale convergence theorem is applicable since $0<Y_{n}<1$, and indeed that the limit random variable $Y$, say, has a beta distribution with parameters $b / c$ and $r / c$ (e.g. Feller (1966), pp. 211, 226). Here we shall be concerned with the rate of convergence of $Y_{n}$ to $Y$.

Write $K_{n}=c /(b+r+(n+1) c)$ and let $F_{n}$ denote the $\sigma$-field generated by $Y_{j}, 0 \leqq j \leqq n$. It is easy to see that, conditional on $Y_{n}$, the distribution of the increment $X_{n+1}=Y_{n+1}-Y_{n}$ is given by

$$
\begin{aligned}
& X_{n+1}=\left(1-Y_{n}\right) K_{n} \quad \text { with probability } Y_{n} \\
& =-Y_{n} K_{n} \quad \text { with probability } 1-Y_{n} \text {. }
\end{aligned}
$$

Then,

$$
E\left(X_{n+1}^{2} \mid F_{n}\right)=Y_{n}\left(1-Y_{n}\right) K_{n}^{2} \sim Y(1-Y) K_{n}^{2} \text { a.s. } \quad \text { as } \quad n \rightarrow \infty
$$

while 
(23) $E X_{n+1}^{2}=K_{n}^{2} E Y_{n}\left(1-Y_{n}\right) \sim K_{n}^{2} E Y(1-Y)=K_{n}^{2} b r(b+r)^{-1}(b+r+c)^{-1}$ as $n \rightarrow \infty$ using dominated convergence since $Y_{n}\left(1-Y_{n}\right) \leqq \frac{1}{4}$. Furthermore,

$$
E\left(X_{n+1}^{4} \mid F_{n}\right)=Y_{n}\left(1-Y_{n}\right) K_{n}^{4}\left\{\left(1-Y_{n}\right)^{3}+Y_{n}^{3}\right\} \leqq \frac{1}{2} K_{n}^{4}
$$

Next, using (23),

$$
s_{n}^{2}=\sum_{j=n}^{\infty} E X_{j}^{2}=\sum_{j=n-1}^{\infty} K_{j}^{2} E Y_{j}\left(1-Y_{j}\right) \sim n^{-1} E Y(1-Y) \quad \text { as } \quad n \rightarrow \infty .
$$

Also, $\sum_{1}^{\infty} S_{n}^{-2}\left\{X_{n}^{2}-E\left(X_{n}^{2} \mid F_{n-1}\right)\right\}$ converges a.s. if, using the Kolmogorov convergence criterion,

$$
\sum_{1}^{\infty} s_{n}^{-4} E X_{n}^{4}<\infty
$$

(Proposition IV-6-2 of Neveu (1970)), and (26) holds by virtue of (24) and (25). Thus, the provisions of Corollary $2[(\mathrm{~b})]$ hold and we shall state the results in terms of normalization using

$$
V_{n}^{2}=\sum_{j=n}^{\infty} E\left(X_{j}^{2} \mid F_{j-1}\right) \sim n^{-1} Y(1-Y) \text { a.s. }
$$

Then, using (25) and (27), $s_{n}^{-2} V_{n}^{2} \stackrel{\text { a.s. }}{\longrightarrow} Y(1-Y) / E Y(1-Y)$, while for $k \geqq n$,

$$
\begin{aligned}
E X_{k}^{2} I\left(\left|X_{k}\right|>\varepsilon s_{n}\right)=\int_{\left\{\left|X_{k}\right|>\varepsilon s_{n}\right\}} X_{k}^{2} d P & <\varepsilon^{-2} s_{n}^{-2} \int_{\left\{\left|X_{k}\right|>\varepsilon s_{n}\right\}} X_{k}^{4} d P \\
& <\frac{1}{2} \varepsilon^{-2} S_{n}^{-2} K_{k}^{4}
\end{aligned}
$$

using (24), so that

$$
s_{n}^{-2} \sum_{k=n}^{\infty} E\left(X_{k}^{2} I\left(\left|X_{k}\right|>\varepsilon s_{n}\right)\right)<\frac{1}{2} \varepsilon^{-2} s_{n}^{-4} \sum_{k=n}^{\infty} K_{k}^{4}=O\left(n^{-1}\right) \quad \text { as } \quad n \rightarrow \infty
$$

and the results of Corollary $1(\mathrm{~b})$ are applicable.

To utilize the iterated logarithm results in Theorem 1 it remains to check Conditions (b)(iii) and (b)(iv). That (b)(iii) holds follows from (24) and (25) since

$$
E\left|X_{n}\right| I\left(\left|X_{n}\right|>\varepsilon s_{n}\right)=\int_{\left\{\left|X_{n}\right|>\varepsilon s_{n}\right\}}\left|X_{n}\right| d P<\varepsilon^{-3} s_{n}^{-3} E X_{n}^{4}=O\left(n^{-5 / 2}\right)
$$

while (b)(iv) also follows from (24) and (25). Theorem 1 thus yields, for example

$$
n^{1 / 2}\left(Y_{n}-Y\right) \stackrel{\mathrm{d}}{\rightarrow}\left[Y^{\prime}\left(1-Y^{\prime}\right)\right]^{1 / 2} N(0,1)
$$

where $Y^{\prime}\left(1-Y^{\prime}\right)$ is distributed as $Y(1-Y)$ and is independent of the $N(0,1)$ random variable, while 


$$
\begin{aligned}
& \limsup _{n \rightarrow \infty} n^{1 / 2}(2 \log \log n)^{-1 / 2}\left(Y_{n}-Y\right)=Y^{1 / 2}(1-Y)^{1 / 2} \text { a.s. } \\
& \liminf _{n \rightarrow \infty} n^{1 / 2}(2 \log \log n)^{-1 / 2}\left(Y_{n}-Y\right)=-Y^{1 / 2}(1-Y)^{1 / 2} \text { a.s. }
\end{aligned}
$$

\section{Acknowledgements}

Thanks are due to J. A. Hartigan for stimulating this research and to B. M. Brown and P. G. Hall for helpful comments.

\section{References}

Athreya, K. B. and Ney, P. E. (1972) Branching Processes. Springer-Verlag, Berlin.

BARBour, A. D. (1974) Tail sums of convergent series of independent random variables. Proc. Camb. Phil. Soc. 75, 361-364.

Billingsley, P. (1968) Convergence of Probability Measures. Wiley, New York.

Chow, Y. S. AND TeICher, H. (1973) Iterated logarithm laws for weighted averages. $Z$. Wahrscheinlichkeitsth. 26, 87-94.

Feller, W. (1966) An Introduction to Probability Theory and its Applications, Vol. II. Wiley, New York.

Hall, P. G. (1977) Martingale invariance principles. Ann. Prob. To appear.

Hall, P. G. And Heyde, C. C. (1976) On a unified approach to the law of the iterated logarithm for martingales. Bull. Austral. Math. Soc. 14, 435-447.

Heyde, C. C. (1968) On almost sure convergence for sums of independent random variables. Sankhyā A30, 353-358.

McLeish, D. L. (1974) Dependent central limit theorems and invariance principles. Ann. Prob. 2, 620-628.

MülLER, D. W. (1968) Verteilungs-Invarianzprinzipien für das starke Gesetz der grossen Zahl. Z. Wahrscheinlichkeitsth. 10, 173-192.

Neveu, J. (1970) Bases Mathématiques du Calcul des Probabilités, 2nd edn. Masson, Paris.

VervaAt, W. (1972) Success Epochs in Bernoulli Trials. Math. Centre Tracts 42, Mathematisch Centrum, Amsterdam.

WhitT, W. (1972) Stochastic Abelian and Tauberian theorems. Z. Wahrscheinlichkeitsth. 22, 251-267. 